\title{
Observations on the Fine Structure of Saccharomyces cerevisiae as Affected by Biotin Deficiency
}

\author{
By B. DIXON ANd A. H. ROSE \\ Department of Microbiology, The University, Newcastle-upon-Tyne
}

(Received 18 November 1963)

\begin{abstract}
SUMMARY
Growth of a biotin-requiring strain of Saccharomyces cerevisiae in a medium containing a suboptimal concentration of biotin caused changes in the fine structure of the yeast as revealed by electron microscopy. The cytoplasmic and vacuolar membranes in yeast grown in unsupplemented biotin-deficient medium were particularly susceptible to polymerization damage and appeared to split along the lipid layer. Similar changes in the appearance of the cytoplasmic membrane were observed in biotin-optimal yeast after treatment with aqueous butanol. Yeast grown in unsupplemented biotin-deficient medium also contained a slightly greater number of large storage granules as compared with exponential phase biotinoptimal yeast. When grown in aspartate-supplemented biotin-deficient medium, the yeast had much thicker cell walls than when grown in unsupplemented biotin-deficient medium. In yeast grown in oleatesupplemented biotin-deficient medium, the cytoplasmic membrane split less extensively along the lipid layer as compared with yeast grown in unsupplemented biotin-deficient medium. Organisms grown in biotindeficient medium supplemented with aspartate + oleate resembled those grown in biotin-deficient medium supplemented with only aspartate in possessing thick cell walls, but otherwise were similar in fine structure to yeast grown in oleate-supplemented biotin-deficient medium. The storage granules in yeast grown in biotin-optimal medium and in unsupplemented biotin-deficient medium stained strongly with Sudan Black B, a lipophilic stain, and their appearance in increased numbers in yeast grown in unsupplemented biotin-deficient medium was accompanied by an increase in the lipid content of the yeast as compared with biotin-optimal yeast. The significance of these changes in fine structure is discussed in relation to changes in chemical composition previously reported to result from an imposition of biotin deficiency in the yeast.
\end{abstract}

\section{INTRODUCTION}

Previous papers from this laboratory have described changes in the chemical composition and enzyme activities of a biotin-requiring strain of Saccharomyces cerevisiae caused by growing the yeast in media containing a suboptimal concentration of biotin. These changes include a decreased synthesis of nucleic acids and total protein as compared with biotin-optimal yeast (Ahmad, Rose \& Garg, 1961), and marked alterations in the amounts of certain enzymes synthesized by the yeast (Ahmad \& Rose, 1962a). Addition of L-aspartate, with or without oleate, to biotindeficient medium caused a partial restoration of nucleic acid and protein synthesis with only a small increase in growth (Ahmad \& Rose, 1962b) and also affected the 
synthesis of certain enzymes as compared with yeast grown in aspartate-free biotin-deficient medium (Ahmad \& Rose, 1962a). Biotin deficiency has also been shown to bring about changes in the permeability properties of yeast, organisms grown in media containing a suboptimal concentration of biotin being much more permeable than biotin-optimal yeast to a wide range of solutes (Rose, 1963). Since biotin is known to function coenzymically in acetyl-CoA carboxylase, an enzyme concerned in fatty acid synthesis (Wakil, 1961), it is possible that the lipoprotein cytoplasmic membrane in biotin-deficient yeast is structurally different from that in biotin-optimal yeast. The biotin-sparing action of certain fatty acids (Williams \& Fieger, 1946; Suomalainen \& Keränen, 1963), and their ability partially to restore the permeability properties of biotin-deficient yeast (Rose, 1963) are presumably due to their action in restoring normal membrane synthesis. Yeast grown in biotin-deficient medium has also been shown to have an altered polysaccharide composition, the organisms containing a greater proportion of glucan and less mannan than biotin-optimal yeast. This alteration in polysaccharide composition was accentuated when the yeast was grown in biotin-deficient medium supplemented with aspartate (Dunwell, Ahmad \& Rose, 1962).

The present paper reports observations on the effect of biotin deficiency on the fine structure of Saccharomyces cerevisiae as revealed by electron microscopy, and shows how certain of these observations can be related to the changes in chemical composition that result from the imposition of this deficiency.

\section{METHODS}

Experimental cultures. The strain of Saccharomyces cerevisiae and the method of maintaining cultures were as described previously by Rose (1960). The yeast was grown in the chemically defined medium of Rose \& Nickerson (1956) using the techniques described by Ahmad et al. (1961). The medium contained either an optimal $\left(8.0 \times 10^{-10} \mathrm{M}\right)$ or a suboptimal $\left(0 \cdot 4 \times 10^{-10} \mathrm{M}\right)$ concentration of D-biotin. In some experiments, biotin-deficient medium was supplemented with L-aspartate $\left(2.0 \times 10^{-3} \mathrm{M}\right)$ and/or oleate (containing $22 \%, \mathrm{w} / \mathrm{w}$, of elaidic acid, the trans isomer of oleic acid; $100 \mu \mathrm{g} . / \mathrm{ml}$.). These biotin-sparing compounds were tested for possible contamination with biotin using the procedure described by Ahmad \& Rose $(1962 b)$. Growth was measured turbidimetrically by determining the extinction of a portion ( $6 \mathrm{ml}$.) of culture or suspension in $\mathrm{M} / 15 \mathrm{KH}_{2} \mathrm{PO}_{4}$ in the Hilger 'Spekker' absorptiometer (Model $\mathbf{H ~ 7 6 0 )}$ ) using neutral green-grey $\mathrm{H} 508$ filters and a water blank. Extinction readings were related to dry wt. of yeast $/ \mathrm{ml}$. by a calibration curve.

Electron microscopy. Yeast was harvested from cultures by centrifugation and was washed once in $\mathrm{M} / \mathbf{1 5} \mathrm{KH}_{\mathbf{2}} \mathrm{PO}_{\mathbf{4}}$ and three times in distilled water. The equivalent of approximately $100 \mathrm{mg}$. dry wt. of yeast was taken in a $15 \mathrm{ml}$. tapered centrifuge tube and prepared for examination in the electron microscope using a modification of the permanganate-uranyl nitrate fixing technique described by Vitols, North \& Linnane (1961). The organisms were fixed by suspending for $10 \mathrm{~min}$. at $0^{\circ}$ in a solution of $\mathrm{KMnO}_{4}(2 \%, \mathrm{w} / \mathrm{v})$ buffered at $\mathrm{pH} 7 \cdot 2$ with veronal acetate, prepared as described by Luft (1956). The yeast was then washed three times in an aqueous solution of uranyl nitrate $(2 \%, w / v)$, the organisms finally remaining in this solu- 
tion for one hour. After suspending in tap water for $15 \mathrm{~min}$., the organisms were dehydrated by serial passage through $25 \%(15 \mathrm{~min}$.) and $70 \%(\mathrm{v} / \mathrm{v})$ ethanol in water (30 min.) and two changes of absolute ethanol $(1 \mathrm{hr}$ each). The dehydrated material was suspended for $\mathbf{1 5} \mathrm{min}$. in a mixture of equal volumes of absolute ethanol and methacrylate monomer mixture (isobutyl and methyl methacrylates, $6+1$ by vol.), followed by suspension for three hours in pure monomer mixture. Finally, the organisms were suspended for $30 \mathrm{~min}$. in a mixture of the monomers $(6+1$ by vol.) which had been partially polymerized with benzoyl peroxide $(2 \%$, w/v) as described by Mercer \& Birbeck (1961). The material was centrifuged and small pieces of the pellet transferred to gelatin capsules (No. 0; Eli Lilley and Co. Ltd., Basingstoke, England) containing partially polymerized methacrylate mixture. The capsules were incubated at $60^{\circ}$ for $48 \mathrm{hr}$ to complete the embedding.

Sections were cut with a Huxley ultra-microtome (manufactured in the Engineering Laboratories, Cambridge University, England) using a glass knife. Only sections showing silver or gold interference patterns (600-1000 $\AA$ thick) were examined. These were floated on to carbon-coated copper grids $(3 \cdot 05 / 3 \cdot 10 \mathrm{~mm} .200 \mathrm{mesh}$; Smethurst High-Light Ltd., Bolton, Lancs., England) and electron micrographs were taken with an Akashi Tronscope (TRS-50) using the $50 \mu$ objective aperture. Photographs were taken at an initial magnification of $\times 4000-16,000$.

Light microscopy. Lipid inclusions in the yeast were demonstrated by staining dried fixed preparations with Sudan Black B according to the procedure of Burdon (1946).

Lipid determinations. Portions containing the equivalent of 100-200 mg. dry wt. yeast that had been washed in $\mathrm{M} / 15 \mathrm{KH}_{2} \mathrm{PO}_{4}$ were transferred to glass-stoppered test tubes $(125 \mathrm{~mm} . \times 20 \mathrm{~mm}$.). After a further washing in water, the organisms were shaken for $30 \mathrm{~min}$. at room temperature with $20 \mathrm{ml}$. of a chloroform-methanol mixture (2:1 by vol.). The solvent mixture was removed after centrifuging and the yeast extracted with a further two portions $(5 \mathrm{ml}$.) of the solvent mixture. The extracts were pooled and filtered quantitatively through a sintered glass filter (Schott u. Gen., Mainz, West Germany; Grade III) into a weighed $50 \mathrm{ml}$. roundbottom flask. The solvents were then removed under reduced pressure at $37^{\circ}$ in an atmosphere of nitrogen, and the flasks and contents dried to constant weight in vacuo over $\mathrm{P}_{2} \mathrm{O}_{5}$. Results are expressed as mg. lipid/100 mg. dry wt. yeast.

\section{RESULTS}

Electron microscopy of thin sections of yeast

Biotin-optimal yeast. Electron micrographs of ultrathin sections of exponential phase $(40 \mathrm{hr}$ ) biotin-optimal yeast (Pl. 1, fig. 1) closely resembled in appearance those previously published by Hirano \& Lindegren (1961) and Vitols et al. (1961). The well-defined cell wall accounted for about one-seventh of the cell diameter and, in certain areas, appeared to show some form of layering. Bud scars showed a greater concentration of electron-dense material than the remainder of the cell wall. The convoluted cytoplasmic membrane beneath the cell wall was similar to the structure described by Vitols et al. (1961), and appeared as a typical unit membrane, approximately $80 \AA$ wide, consisting of two electron-dense layers of protein separated by an electron-transparent lipid layer (Robertson, 1959; Pl. 1, fig. 2). In 
electron micrographs of sections of exponential phase biotin-optimal yeast, there was only very rarely a gap between the cytoplasmic membrane and the cell wall. The main structures in the cytoplasm of biotin-optimal yeast were the lobular vacuoles, which contained electron-dense particles probably of polyphosphate, and the electron-dense nucleus; both the nucleus and the vacuoles appeared to be bounded by membranes. The highly electron-transparent areas probably represent storage granules. Substantially the same fine structure was observed in earlier exponential phase and stationary phase biotin-optimal yeast.

Biotin-deficient yeast. Yeast grown for $120 \mathrm{hr}$ (exponential phase) in unsupplemented biotin-deficient medium (Ahmad \& Rose, 1962 b) showed certain well-marked differences in fine structure as compared with biotin-optimal yeast (Pl. 1, figs. 3 and 4). In general these organisms seemed to be more susceptible than biotin-optimal yeast to polymerization damage during preparation for electron microscopy. Sections of biotin-deficient yeast rarely showed a cytoplasmic membrane similar in structure to that in biotin-optimal yeast, and there was always a gap between the cell wall and the cytoplasm. A thin electron-dense layer, probably of protein, appeared on the inner edge of the cell wall and on the outer edge of the cytoplasm and, at certain points, these layers appeared to be joined by thin strands of electron-dense material (Pl. 1, fig. 3). This appearance suggested that the cytoplasmic membrane in yeast grown in unsupplemented biotin-deficient medium had split along the lipid layer probably when the yeast was subjected to the stress of preparation for electron microscopy. Also, certain of the cytoplasmic organelles in these organisms seemed to be structurally different from those in biotin-optimal yeast, and the vacuolar membranes often appeared to have severed in the same way as the cytoplasmic membrane (Pl. 1, fig. 4). Yeast grown in unsupplemented biotindeficient medium also contained a slightly greater number of larger storage granules as compared with biotin-optimal yeast, although it is possible that some of these electron-transparent areas represent space formally occupied by vacuoles the contents of which were released after the vacuolar membrane had severed.

The most striking feature of sections of yeast grown for $72 \mathrm{hr}$ (exponential phase) in aspartate-supplemented biotin-deficient medium (Ahmad \& Rose, 1962b) was the appearance of the cell wall, which, although it varied somewhat in thickness, was in general much thicker than the walls of biotin-optimal yeast or of yeast grown in unsupplemented biotin-deficient medium (Pl. 2, fig. 5). The walls also showed a marked layering and distortions not seen in these other yeasts. In some sections, the layers of the wall appeared to have separated under the stress imposed during preparation for electron microscopy. This multiple layering of the walls was particularly noticeable in organisms that had failed to separate from mother cells (Dunwell et al. 1962). Yeast grown in aspartate-supplemented biotin-deficient medium resembled that grown in unsupplemented biotin-deficient medium in possessing a gap beneath the cell wall and in lacking an intact cytoplasmic membrane. The cytoplasmic contents of aspartate-grown biotin-deficient yeast were more electron-dense than those in biotin-optimal yeast or in yeast grown in unsupplemented biotin-deficient medium, and the vacuoles and granules were smaller in size and less numerous.

In yeast grown for $120 \mathrm{hr}$ (exponential phase) in biotin-deficient medium supplemented with oleate (Ahmad \& Rose, 1962 b; Pl. 2, fig. 6), the cell-wall structure 
resembled that in biotin-optimal yeast and in yeast grown in unsupplemented biotin-deficient medium. The most interesting feature of these organisms grown in the presence of oleate was the appearance of the cytoplasmic membrane. Again, there was a gap between the cell wall and the cytoplasm, but the cytoplasmic membrane did not appear to have split so extensively along the lipid layer as in yeast grown in unsupplemented biotin-deficient medium. Instead, it appeared to remain intact, at least at certain points, and to adhere to the cytoplasm. The cytoplasm did not differ greatly in appearance from that in yeast grown in unsupplemented biotin-deficient medium, although the membranes surrounding the vacuoles and nucleus were more distinct.

When yeast was grown for $72 \mathrm{hr}$ (exponential phase) in biotin-deficient medium supplemented with aspartate + oleate (Ahmad \& Rose, 1962 b; Pl. 2, fig. 7), the cell wall resembled in appearance that in yeast grown in biotin-deficient medium supplemented with only aspartate ( $\mathrm{Pl}$. 2, fig. 5). Apart from being rather more electron-dense, the cytoplasm and the cytoplasmic membrane were similar to that seen in sections of yeast grown in oleate-supplemented biotin-deficient medium.

Effect of butanol treatment. Treatment with aqueous butanol is known to break the osmotic barrier in many micro-organisms (Mitchell \& Moyle, 1956) and it has been shown (Rose, 1963) that the osmotic behaviour of biotin-optimal yeast treated with aqueous butanol resembles that of untreated biotin-deficient yeast. In view of the difference in structure of the cytoplasmic membrane in biotin-optimal yeast (Pl. 1, fig. 2) and in yeast grown in unsupplemented biotin-deficient medium (Pl. 1, fig. 3), it seemed of interest to compare the effect of butanol treatment on the structure of the membrane in biotin-optimal yeast with the membrane in untreated biotin-deficient yeast.

Washed biotin-optimal yeast $(40 \mathrm{hr})$ was treated with a solution of $5 \%(\mathrm{v} / \mathrm{v})$ $n$-butanol in $\mathrm{M} / 15 \mathrm{KH}_{2} \mathrm{PO}_{4}(\mathrm{pH} \mathrm{4} \cdot 5)$ as described by Rose (1963). The yeast was then removed by centrifugation and prepared for examination in the electron microscope as described under Methods. Examination of electron micrographs of thin sections of the butanol-treated yeast showed that, with larger organisms, the treatment so altered the structure of the yeast that the cell wall ruptured during preparation for electron microscopy. Smaller organisms, however, remained intact following treatment with butanol (Pl. 2, fig. 8), and the cytoplasmic membrane resembled in appearance the split membranes seen in sections of untreated biotindeficient yeast (Pl. 1, fig. 3). Certain of the cytoplasmic organelles in the butanoltreated biotin-optimal yeast were also altered in appearance.

\section{Composition of the storage granules}

One particularly interesting result from this study on the effect of biotin deficiency on the fine structure of the yeast was the appearance in yeast grown in unsupplemented biotin-deficient medium (PI. 1, fig. 3) of an increased number of large electron-transparent areas, at least some of which probably represent storage granules. Previous workers who have observed these structures in biotin-optimal yeast have suggested that they consist of glycogen, which is the characteristic storage material in yeast, or of lipid. But the glycogen contents of biotin-optimal yeast and of yeast grown in unsupplemented and aspartate-supplemented biotindeficient medium are not significantly different (Dunwell et al. 1962), which suggests 
that the additional storage granules which appeared in yeast grown in unsupplemented biotin-deficient medium do not represent extra deposits of glycogen. It was shown furthermore that the storage granules in biotin-optimal yeast and in yeast grown in unsupplemented biotin-deficient medium were probably not of poly- $\beta$-hydroxybutyrate (PHB). After the granules had been released from the organisms by treatment with alkaline sodium hypochlorite ('Domestos', commercial preparation; Law \& Slepecky, 1961), they were shown to be freely soluble in ether, a solvent in which PHB is not soluble (Williamson \& Wilkinson, 1958). However, when dried fixed preparations of the yeasts were examined, these granules were shown to stain strongly with Sudan Black B, a lipophilic stain (Burdon, 1946), and this prompted an examination of the lipid content of the yeast grown under different conditions of biotin deficiency.

Table 1. Effect of biotin deficiency on the lipid content of the yeast

\begin{tabular}{|c|c|c|}
\hline Medium & $\begin{array}{l}\text { Age of } \\
\text { culture } \\
\text { (hr) }\end{array}$ & $\begin{array}{c}\text { Lipid content* } \\
\text { (mg. lipid/100 mg. dry wt. } \\
\text { yeast) }\end{array}$ \\
\hline Biotin-optimal & 40 & $3 \cdot 43 \pm 0 \cdot 18$ \\
\hline Biotin-deficient & 120 & $4 \cdot 27 \pm 0.35$ \\
\hline Biotin-deficient + aspartate & 72 & $2 \cdot 96 \pm 0 \cdot 21$ \\
\hline Biotin-deficient + oleate & 120 & $3 \cdot 40 \pm 0 \cdot 33$ \\
\hline Biotin-deficient + aspartate +oleate & 72 & $3 \cdot 97 \pm 0 \cdot 29$ \\
\hline
\end{tabular}

* Each figure represents the average of at least seven determinations.

The data (Table 1) show that organisms grown in unsupplemented biotindeficient medium contained rather more lipid than biotin-optimal yeast. The lipid content of organisms grown in the aspartate-supplemented biotin-deficient medium was considerably lower than that of yeast grown in unsupplemented biotin-deficient medium and also lower than that of biotin-optimal yeast. Organisms grown in biotin-deficient medium supplemented only with oleate contained approximately the same amount of lipid as biotin-optimal yeast, and organisms grown in biotindeficient medium supplemented with both oleate + aspartate contained rather more lipid than did yeast grown in biotin-deficient medium supplemented with oleate alone. Since an apparent increase in lipid content might be caused by adsorption of fatty acid on to yeast grown in oleate-supplemented media, control experiments were carried out in which the lipid contents of yeast grown for $120 \mathrm{hr}$ in biotindeficient medium or for $\mathbf{7 2} \mathbf{~ h r}$ in aspartate-supplemented biotin-deficient medium were determined after the organisms had been harvested and suspended for $\mathbf{3 0}$ min. in the corresponding medium supplemented with oleate. However, with neither of these yeasts was there a detectable increase in the apparent lipid content of the organisms following this treatment.

\section{DISCUSSION}

Several of the changes in chemical composition of Saccharomyces cerevisiae that are known to result from an imposition of biotin deficiency can be related to changes in the fine structure of the yeast. For example, the structure of the cytoplasmic and vacuolar membranes in yeast grown in unsupplemented biotin-deficient 
medium appears to be altered to such an extent that the membranes become damaged during preparation of the specimen for electron microscopy. This behaviour can be correlated with increased permeability of biotin-deficient yeast to a wide range of solutes (Rose, 1963). The cytoplasmic membrane in yeast grown in unsupplemented biotin-deficient medium tends to split along the lipid layer, which suggests that this component of the lipoprotein membrane is structurally different from its counterpart in the membrane of biotin-optimal yeast. This conclusion is supported by the finding that, when synthesis of protein, the other main constituent of the lipoprotein membrane, is restored by growing the yeast in a biotin-deficient medium supplemented with aspartate, neither the fine structure of the membrane nor its permeability properties (Rose, 1963) resembled those of biotin-optimal yeast. But addition of oleic acid (including $22 \%, \mathrm{w} / \mathrm{w}$, elaidic acid) to the biotindeficient medium causes a partial restoration of the osmotic behaviour of the yeast (Rose, 1963) and also leads to changes in the fine structure of the cytoplasmic membrane. The inability of these fatty acids to restore completely the permeability properties and fine structure of the membrane may be due to the production of a membrane of abnormal composition in yeast grown in oleate-supplemented biotindeficient medium. Thus, it has been shown that the fatty acid composition of the total lipid in a biotin-requiring strain of Lactobacillus delbrueckii varies according to whether growth in a biotin-deficient medium was promoted by biotin or by various biotin-sparing unsaturated fatty acids (Hofmann, Henis \& Panos, 1957).

The electron micrographs described in the present paper also provide further information on the changes in cell wall composition that result from growth of the yeast in a biotin-deficient medium supplemented with aspartate. Dunwell et al. (1962) reported that yeast grown in this medium contained an increased amount of total polysaccharide with a greater proportion of glucan and less mannan than biotin-optimal yeast or yeast grown in unsupplemented biotin-deficient medium. In addition, the organisms tended to remain attached in clumps. The electron micrographs show that this increased glucan synthesis leads to the formation of much thicker cell walls as compared with yeast grown in aspartate-free biotindeficient medium. They also show that, in yeast grown in aspartate-supplemented biotin-deficient medium, over-production of cell wall material tends occasionally to be localized at certain points on the surface of the protoplast. It appears, too, that the clumping of these organisms is due to a failure to complete the division process by formation of a cross-septum between the walls of the mother and daughter cells. Little is known of the nature of the enzymes involved in the synthesis of cell wall material in yeast or of the ways in which this synthesis is controlled. It is possible, however, that biotin deficiency may lead to a breakdown in these regulatory mechanisms just as it can alter the mechanisms regulating synthesis of the enzyme ornithine carbamoyltransferase in this yeast (Dixon \& Rose, 1964).

The appearance in yeast grown in unsupplemented biotin-deficient medium of an increased number of large storage granules, at least some of which may be of lipid, was somewhat surprising in view of the well established biotin-sparing action of fatty acids (Hofmann, O'Leary, Yoho \& Liu, 1959; Williams \& Fieger, 1946). However, Croom \& McNeill (1961) have reported that growth of Lactobacillus arabinosus under conditions of biotin deficiency led to a decrease in the amount of unsaturated fatty acids in the bacterium and an increase in the amount of the 
saturated fatty acid palmitate although the opposite effect was reported for a biotin-requiring strain of Escherichia coli. Imposition of biotin deficiency in another strain of Saccharomyces cerevisiae (Suomalainen \& Keränen, 1963) has been reported to lead to synthesis of a greater proportion of shorter chain fatty acids as compared with biotin-optimal yeast, so that it is possible that the increased synthesis of lipid in our strain of yeast grown in unsupplemented biotin-deficient medium may be due mainly to over-production of shorter chain fatty acids.

The authors wish to thank Miss Judith Hall and Miss Margaret Bolam for valuable technical assistance during this study, and Mr E. H. Boult of the School of Chemistry, The University, Newcastle-upon-Tyne, for kindly providing facilities for electron microscopy. One of us (B.D.) is indebted to the Luccock Research Fund for financial assistance.

\section{REFERENCES}

Ahmad, F. \& Rose, A. H. (1962a). The role of biotin in the regulation of enzyme synthesis in yeast. Arch. Biochem. Biophys. 97, 302.

Ahmad, F. \& Rose, A. H. (1962b). Effect of biotin-sparing substances on growth of biotindeficient Saccharomyces cerevisiae and on the synthesis of nucleic acids and protein. $J$. gen. Microbiol. 28, 147.

Ahmad, F., Rose, A. H. \& Garg, N. K. (1961). Effect of biotin deficiency on the synthesis of nucleic acids and protein by Saccharomyces cerevisiae. J. gen. Microbiol. 24, 69.

Burdon, K. L. (1946). Fatty material in bacteria and fungi revealed by staining dried fixed slide preparations. J. Bact. 52, 665 .

Croom, J. A. \& McNeill, J. J. (1961). The long chain fatty acids of certain biotinrequiring bacteria. Bact. Proc. p. 170.

Dixon, B. \& Rose, A. H. (1964). On the synthesis of ornithine carbamoyltransferase in biotin-deficient Saccharomyces cerevisiae. J. gen. Microbiol. 34, 229.

Dunwell, J. L., Ahmad, F. \& Rose, A. H. (1962). Changes in the polysaccharide composition of yeast resulting from biotin deficiency. Biochim. biophys. Acta, 51, 604.

Hirano, T. \& Lindegren, C. C. (1961). Electron microscopy of mitochondria in Saccharomyces. J. ultrastruct. Res. 5, 321.

Hofmann, K., Henis, D. B. \& Panos, C. (1957). Fatty acid interconversions in lactobacilli. J. biol. Chem. 228, 349.

Hofmann, K., O'Leary, W. M., Yoho, C. W. \& LiU, T.-Y. (1959). Further observations on lipid stimulation of bacterial growth. J. biol. Chem. 234, 1672.

LAw, J. H. \& Slepecky, R. A. (1961). Assay of poly- $\beta$-hydroxybutyric acid. J. Bact. 82, 33.

LuFt, J. H. (1956). Permanganate-a new fixative for electron microscopy. J. biophys. biochem. Cytol. $2,799$.

Mercer, E. H. \& Birbeck, M. S. C. (1961). Electron Microscopy. Oxford: Blackwell.

Mrtcheld, P. D. \& Moyle, J. M. (1956). Osmotic function and structure in bacteria. In Bacterial Anatomy. Symp. Soc. gen. Microbiol. 6, 150.

Robertson, J. D. (1959). In The Structure and Function of Subcellular Components. Biochem. Soc. Symp. 16, 3.

Rose, A. H. (1960). Excretion of nicotinic acid by biotin-deficient Saccharomyces cerevisiae. J. gen. Microbiol. 23, 143.

Rose, A. H. (1963). On the osmotic behaviour of Saccharomyces cerevisiae as affected by biotin deficiency. J. gen. Microbiol. 31, 151.

Rose, A. H. \& Nickerson, W. J. (1956). Secretion of nicotinic acid by biotin-dependent yeasts. J. Bact. 72, 324. 
Journal of General Microbiology, Vol. 35, No. 3

Plate 1
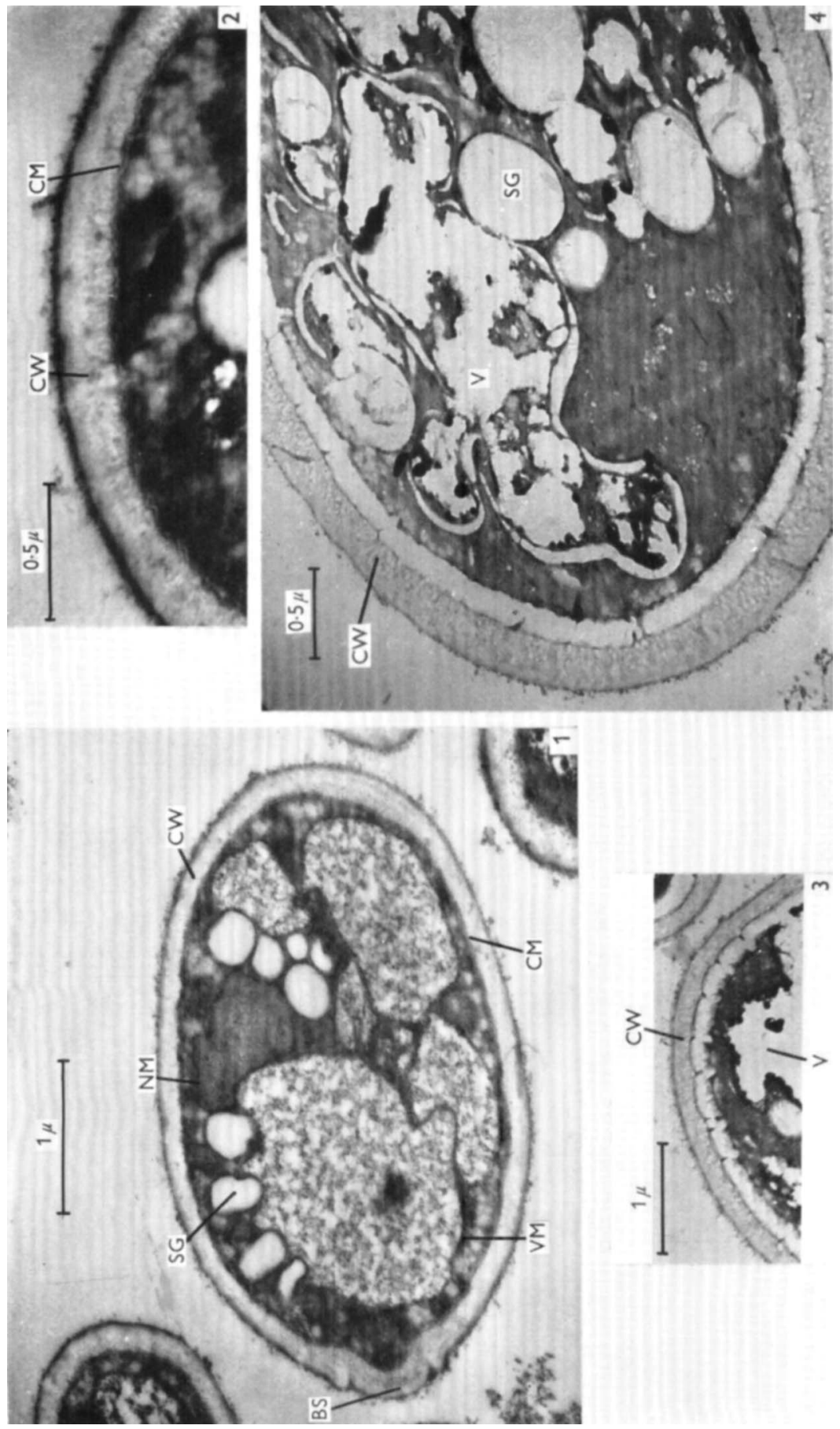

B. DIXON AND A. H. ROSE

(Facing p. 418) 

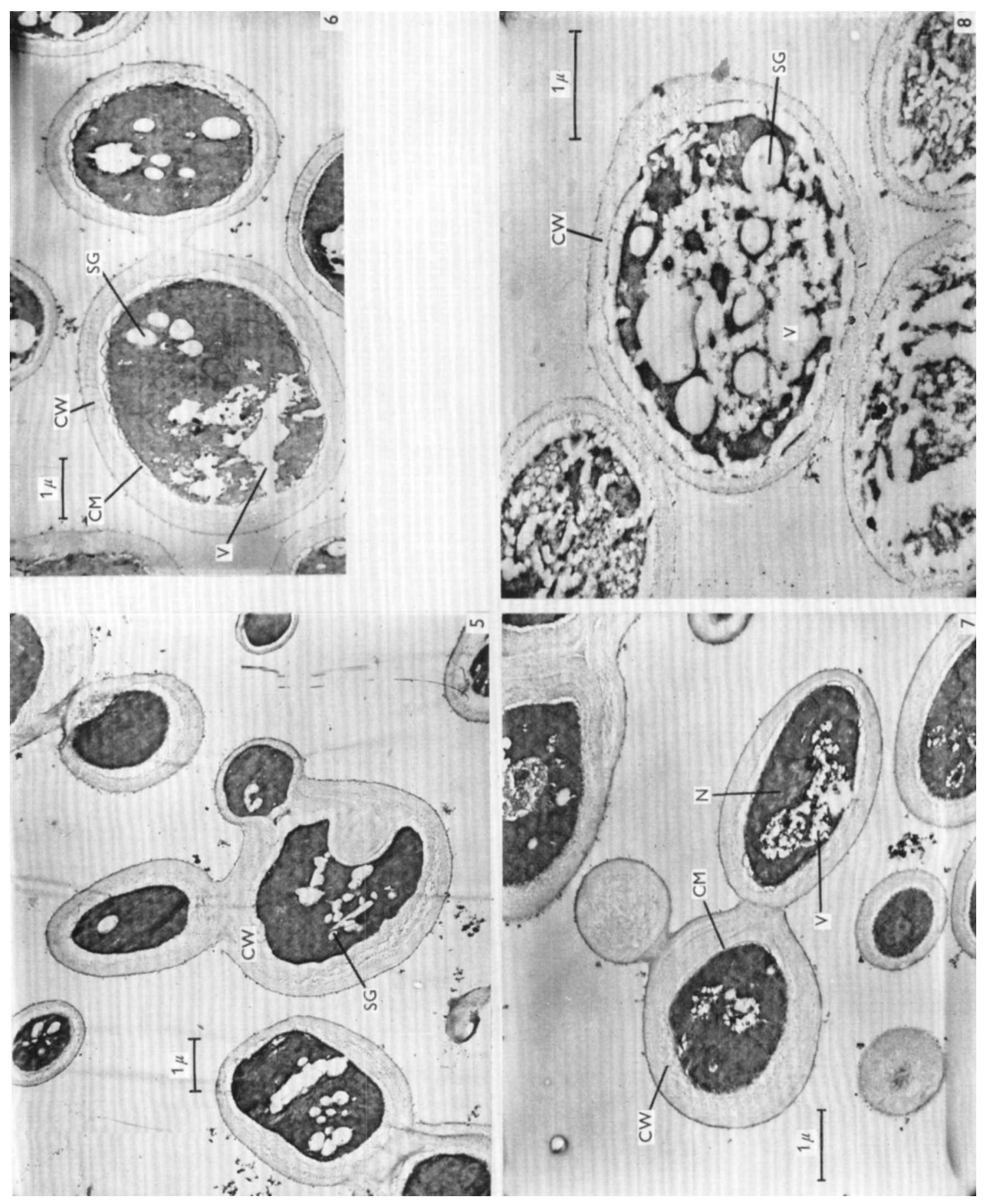

B. DIXON AND A. H. ROSE 
Suomalainen, H. \& Keränen, A. J. A. (1963). The effect of biotin deficiency on the synthesis of fatty acids by yeast. Biochim. biophys. Acta, 70, 493.

Vitols, E., North, R. J. \& Linnane, A. W. (1961). Studies on the oxidative metabolism of Saccharomyces cerevisiae. 1. Observations on the fine structure of the yeast cell. J. biophys. biochem. Cytol. 9, 689.

WAKIL, S. J. (1961). Mechanisms of fatty acid synthesis. J. lipid Res. $2,1$.

Wiluiams, V. R. \& Fieger, E. A. (1946). Oleic acid as a growth stimulant for Lactobacillus casei. J. biol. Chem. 166, 335.

Williamson, D. H. \& Wilkinson, J. F. (1958). The isolation and estimation of poly- $\beta$ hydroxybutyrate inclusions of Bacillus species. J. gen. Microbiol. 19, 198.

\section{EXPLANATION OF PLATES}

Saccharomyces cerevisiae, electron micrographs. BS, bud scar; CM, cytoplasmic membrane; $\mathrm{CW}$, cell wall ; N, nucleus; NM, nuclear membrane; SG storage granule; V, vacuole ; VM, vacuolar membrane.

\section{Plate 1}

Fig. 1. Section through an organism from a $40 \mathrm{hr}$ culture of the yeast grown in biotin-optimal medium.

Fig. 2. Part of a section of a biotin-optimal yeast as in Fig. 1, enlarged to show the structure of the cell wall and the cytoplasmic membrane.

Fig. 3. Part of a section through an organism from a 120 hr culture of the yeast grown in unsupplemented biotin-deficient medium.

Fig. 4. Section through an organism from a $120 \mathrm{hr}$ culture of the yeast grown in unsupplemented biotin-deficient medium showing the structure of the vacuoles and vacuolar membranes.

\section{Plate 2}

Fig. 5. Section through organisms from a $72 \mathrm{hr}$ culture of the yeast grown in aspartatesupplemented biotin-deficient medium.

Fig. 6. Section through organisms from a $120 \mathrm{hr}$ culture of the yeast grown in oleatesupplemented biotin-deficient medium.

Fig. 7. Section through organisms from a $72 \mathrm{hr}$ culture of the yeast grown in biotin-deficient medium supplemented with aspartate + oleate.

Fig. 8. Section through an organism from a $40 \mathrm{hr}$ culture of the yeast grown in biotin-optimal medium. The yeast was treated with $5 \%(\mathrm{v} / \mathrm{v}) n$-butanol in $\mathrm{M} / 15 \mathrm{KH}_{2} \mathrm{PO}_{4}$ before preparation for electron microscopy. 